\title{
Voneinander Lernen - Anerkennung und Anrechnung im internationalen Vergleich
}

\author{
Nico Sturm • Helmar Hanak
}

Eingegangen: 26. Juni 2015 / Angenommen: 30. Oktober 2015 / Online publiziert: 2. Dezember 2015

(C) Die Autor(en) 2015. Dieser Artikel ist auf Springerlink.com mit Open Access verfügbar.

Zusammenfassung Das Lebenslange Lernen zielt als eines der zentralen Themen des Bologna-Prozesses unter anderem auf die horizontale und vertikale Durchlässigkeit in einem vernetzten Bildungssystem ab, dass vielfältige Bildungswege für unterschiedliche Zielgruppen ermöglichen soll. Damit gewinnt auch die Thematik der Anerkennung und Anrechnung außerhochschulisch erworbener Kompetenzen an Bedeutung. Trotz unterschiedlicher Bemühungen in den vergangenen zehn Jahren sind bundeseinheitliche Rahmenbedingungen für das Vorgehen auf diesem Feld bis heute nur schwach ausgeprägt.

Anhand einer Verfahrensgegenüberstellung wird der Frage nachgegangen, welche Adaptierungspotentiale die französische Systematik mit ihren Modellen zur Anerkennung und Anrechnung von außerhochschulisch erworbenen Kompetenzen für die deutsche Hochschullandschaft bietet. Dazu findet eine Betrachtung auf zwei Ebenen statt. Zum einen werden die Möglichkeiten der Zulassung zu einem Studienprogramm, für Bewerbende ohne formale Hochschulzugangsberechtigung, durch den Einsatz außerhochschulisch erworbener Kompetenzen verglichen. Zum anderen werden die Möglichkeiten der Verkürzung des zu studierenden Workloads durch die Berücksichtigung bereits vorhandener Kompetenzen gegenüber gestellt. Aus der Gesamtbetrachtung der dargestellten Verfahren sollen abschließend Adaptierungspotentiale abgeleitet werden.

\footnotetext{
N. Sturm, M.A. (囚)

Technische Hochschule Mittelhessen,

Wetzlar, Deutschland

E-Mail: nico.sturm@uni-marburg.de

H. Hanak, M.A.

Servicestelle Offene Hochschule Niedersachsen gGmbH,

Hannover, Deutschland

E-Mail: helmar.hanak@servicestelle-ohn.de
} 
Schlüsselwörter Systemvergleich · Adaptierung · Anrechnung · Kompetenzen · Durchlässigkeit

Abstract Lifelong Learning, being one of the key issues of the Bologna Process, aims at horizontal and vertical permeability of interdependent education systems in order to be accessible to many target groups. Hence, the recognition and crediting of qualifications achieved outside of the academic realm has become increasingly more important. Despite numerous efforts within the past 10 years national standards regarding this issue are not sufficiently developed at this point.

The comparison of the different procedures of France and Germany in this matter is followed by an attempt to answer the question in what ways the French system of recognition and crediting of qualifications achieved outside of the academic realm can serve as a model for Germany. There will be an analysis at two levels. On the one hand, the paper will focus on admission requirements and examine the possibilities of admission without an official university entrance qualification and whether additional non-academic competencies are recognized. On the other hand, the different ways of reducing the required workload by acknowledging previously obtained competencies will be compared. As a result possible adaptations will be inferred from the presented procedures and analyses.

Keywords comparison of the different procedures $\cdot$ adaptation $\cdot$ recognition $\cdot$ competences $\cdot$ permeability

\section{Einleitung}

In Zeiten, in denen der demografische Wandel die Gesellschaftsstrukturen und der Fachkräftemangel den Arbeitsmarkt nachhaltig beeinflussen, kommt der Öffnung der Hochschulen ${ }^{1}$ für neue Zielgruppen eine zentrale Bedeutung zu. Um diese Öffnung weiter voranzubringen, haben die politisch Verantwortlichen in Deutschland in den vergangenen Jahren entsprechende rechtliche Rahmenbedingungen geschaffen, damit eine belastbare Grundlage für die Anrechnung ${ }^{2}$ außerhochschulisch erworbener Kompetenzen zur Verfügung steht (KMK 2002, 2008, 2009).

Des Weiteren unterstützt der Fachdiskurs in der einschlägigen Literatur die Entwicklungen. So werden beispielsweise durch Freitag et al. (2015) aktuelle GoodPractice-Beispiele und Ansätze zur Beratung aufgegriffen. Schäfer et al. (2015) widmen sich der Darstellung von Wegen zur akademischen Qualifizierung im Sozialund Gesundheitssystem und Seger und Waldeyer (2015) geben konkrete Hinweise zur qualitätsgesicherten Anrechnung von außerhochschulisch erworbene Kompetenzen. Gleichzeitig wurden durch Projekte wie beispielsweise die ANKOM-Initiative (2005-2014) oder der Bund-Länder-Wettbewerb Aufstieg durch Bildung: Offene Hochschulen (2011-2020) entsprechende Verfahren ins Leben gerufen. Resultierend

\footnotetext{
${ }^{1}$ Im Folgenden sind mit „Hochschulen“ alle Universitäten sowie Hochschulen angewandter Wissenschaften (of applied science), Fachhochschulen und sonstige fachlichen Hochschulen gemeint.

${ }^{2}$ Siehe zur Begriffsbestimmung auch www.hrk-nexus.de/meta/glossar/quelle/default/eintrag/anrechnung-121.
} 
hieraus sind unterschiedliche Modelle (individuelle, pauschale und kombinierte) zur Anrechnung formaler, non-formaler sowie informeller Kompetenzen entwickelt, implementiert und umgesetzt worden. Aus diesen Entwicklungsarbeiten der vergangenen zehn Jahre ist ein breites Portfolio zweckdienlicher und qualitätsgesicherter Methoden hervorgegangen, die auch andere Hochschulen nach ihren jeweiligen Bedürfnissen adaptieren könnten. Trotz dieser entwickelten und erprobten Verfahren, sowie der eindeutigen Aufforderung,

von den bestehenden Möglichkeiten der Anrechnung Gebrauch zu machen und Verfahren und Kriterien für die Anrechnung außerhalb des Hochschulwesens erworbener Kenntnisse und Fähigkeiten in den jeweiligen Prüfungsordnungen zu entwickeln (KMK 2008, S. 4),

haben sich bisher weder bundeslandspezifische noch ein bundeseinheitliches Verfahren etabliert.

Mit diesem Beitrag wird im Rahmen eines systematischen Vergleichs der Frage nachgegangen, welche Adaptierungspotenziale Frankreich mit seinen Verfahren zur Anrechnung außerhochschulisch erworbener Kompetenzen für die deutsche Hochschullandschaft vor diesem Hintergrund bietet.

Frankreich wurde explizit ausgewählt, da es mit der Anrechnung außerhochschulisch erworbener Kompetenzen bis hin zur Vergabe ganzer Studienabschlüsse sehr weitreichend ist. Diese deutlich liberalere Haltung in Bezug auf Anrechnungsfragen im Vergleich zum deutschen Hochschulsystem kann in Zeiten steigender Mobilität im europäischen Binnenmarkt einen erhöhten Wettbewerbsvorteil darstellen. Zudem weisen die französischen Verfahren eine weitere Konvergenz zum deutschen dahingehend auf, dass auch hier zwischen Anrechnung mit dem Ziel der Zulassung zum Studium (Validation des Acquis Professionnels - VAP) und Anrechnung mit dem Ziel der Verkürzung des zu studierenden Workloads (Validation des Acquis de l'Expérience - VAE) durch die Berücksichtigung von Kompetenzen, die sowohl im beruflichen als auch im privaten und/oder im freiwilligen Bereich erworben wurden, unterschieden wird. Durch die föderalistischen Strukturen in der Bundesrepublik Deutschland mit Blick auf die Organisation des Bildungssystems ergibt sich ein deutlicher Kontrast zu dem stark zentralistischen Staatsaufbau in Frankreich. Ein signifikanter Unterschied in der Betrachtung der beiden Länder ist in den sehr unterschiedlichen bildungspolitischen Rand- und Rahmenbedingungen zu sehen.

Im Folgenden werden zunächst die Systematiken zur Anrechnung der beiden Länder in Bezug auf die Dimensionen Zulassung und Verkürzung gegenübergestellt, um abschließend in einem Fazit mögliche Adaptierungspotenziale für das deutsche Hochschulsystem aufzeigen zu können.

\section{Vergleich der Systeme im Kontext der Zulassung zu einem Studium}

Zunächst werden die Systeme ${ }^{3}$ der Anrechnung außerhochschulisch erworbener Kompetenzen mit dem Ziel der Zulassung zu einem Hochschulprogramm für Bewer-

\footnotetext{
${ }^{3}$ Im Folgenden sind mit dem Begriff „System“ die Verfahren und Systematiken zur Anrechnung außerhochschulisch erworbener Kompetenzen gemeint.
} 
bende dargestellt, die über keine allgemeine oder fachgebundene HZB verfügen. Dabei werden schwerpunktmäßig die Aspekte Finanzen, Beratung und Vernetzung betrachtet.

2.1 Anrechnung als Instrument zur Zulassung zu einem Studium ohne HZB in Frankreich (VAP)

Die Möglichkeit, die sogenannte VAP zu nutzen, um zu einem Hochschulstudium zugelassen zu werden, ohne über eine formale HZB zu verfügen, wurde in Frankreich bereits im Jahr 1985 per Dekret verankert (vgl. Französisches Bildungsministerium 1985). Die Voraussetzungen zur Teilnahme an diesem Verfahren bestehen darin, dass Bewerbende eine Ausbildung seit mindestens zwei Jahren erfolgreich abgeschlossen haben müssen und mindestens 20 Jahre alt sind.

Zur Finanzierung dieses Verfahrens müssen die Antragstellenden eine Gebühr entrichten. Die Höhe der Kosten ist dabei abhängig von den Vorgaben der jeweiligen Hochschule. Der weitgehend standardisierte Verfahrensablauf sieht vor, dass Bewerbende einen Antrag auf Zulassung (la recevabilité) ausfüllen und diesen an der Hochschule, an der das Studium aufgenommen werden soll, nach einem durch die Hochschule definierten Zeitplan einreichen. Nach vollständiger und erfolgreicher Antragstellung erhalten die Bewerbenden weiterführende Unterlagen, welche sie mit Angaben zu professionellen, persönlichen und eventuellen universitären Erfahrungen vervollständigen müssen.

Ziel dieser Dokumentation ist es, über die Darstellung des eigenen Wissens und der bereits erworbenen Kompetenzen die Anschlussfähigkeit an das angestrebte Studium zu verdeutlichen. Dabei soll auf die Studieninhalte und Kompetenzbeschreibungen des Modulhandbuchs des angestrebten Studiengangs Bezug genommen werden. Darüber hinaus werden die Bewerbenden von der den Antrag bearbeitenden Hochschule dazu angehalten, ihre persönlichen und beruflichen Werdegänge aufzuzeigen und das Niveau zu definieren, auf das sie eingestuft werden wollen (Zertifikat, Bachelor, Master). Die genauen Inhalte der Bewerbungsunterlagen werden dabei von der jeweiligen Hochschule festgelegt und den Bewerbenden mitgeteilt (vgl. Französisches Bildungsministerium 1985: Artikel 6).

Nach dem Einreichen der vollständigen Unterlagen durch die Antragstellenden werden diese durch eine pädagogische Kommission geprüft, um festzustellen, ob die Bewerbenden das Studium beginnen und in welches Niveau des Studiums sie aufgrund ihrer bereits vorhandenen Kompetenzen eingestuft werden können. Bei Unklarheiten besteht die Möglichkeit, die Antragstellenden zusätzlich von der Kommission zu einem Interview einzuladen. Über die Feststellung der Eignung zum Studium hinaus kann dieses Interview auch dazu genutzt werden, die Motivation für das Aufnehmen des Studienangebots zu erörtern (vgl. ebd.: Artikel 7).

Die pädagogische Kommission wird vom Präsidenten der Hochschule konstituiert. Dabei ist vorgeschrieben, dass der Vorsitz der Kommission durch eine Professur der jeweiligen Hochschule besetzt sein muss. Des Weiteren muss sich die Kommission aus zwei weiteren Lehrenden der Fachrichtung und einem Lehrenden aus der beruflichen Weiterbildung des entsprechenden Fachgebiets zusammensetzen. Die Entscheidung zur Aufnahme der Bewerbenden trifft der Präsident beziehungs- 
weise die Präsidentin der Hochschule in Absprache mit der Kommission. Bei nicht zulassungsbeschränkten Studienangeboten erfolgt die administrative Einschreibung unmittelbar nach Prüfung der Bewerbung durch die Kommission. Handelt es sich um einen zulassungsbeschränkten Studiengang, kann eine weitere formale Prüfung der Bewerbung erfolgen (vgl. ebd.: Artikel 8).

Insgesamt kann das gesamte Bewerbungsverfahren von der ersten Antragstellung bis zur Einschreibung in die Hochschule über einen Zeitraum von ein bis sechs Monaten andauern.

\subsection{Anrechnung als Instrument zur Zulassung zu einem Studium ohne HZB in Deutschland}

In Deutschland hat die KMK im Jahr 2009 einen ersten Anstoß zur Vereinheitlichung der bis dahin aufgrund der föderalistischen Struktur der Bundesrepublik sehr pluralistischen Regelungen der Hochschulzulassung ohne Abitur gegeben. Somit verfügen unter anderem Meister im Handwerk (§ 45, 51a, 122 Gesetz zur Ordnung des Handwerks - HWO), Inhaber und Inhaberinnen von Fortbildungsabschlüssen ( $\S \S 53,54$ Berufsbildungsgesetz - BBiG), sofern die Lehrgänge mindestens 400 Unterrichtsstunden umfassen, von Abschlüssen von Fachschulen entsprechend der „Rahmenvereinbarung über Fachschulen“ (vgl. KMK 2014a) und von Abschlüssen vergleichbarer landesrechtlicher Fortbildungsregelungen für Berufe im Gesundheitswesen sowie im Bereich der sozialpflegerischen und sozialpädagogischen Berufe über eine HZB (vgl. KMK 2014b).

Unter bestimmten Voraussetzungen können auch beruflich qualifizierte Bewerbende eine fachgebundene HZB erlangen. Hierzu müssen die Antragstellenden ein Eignungsfeststellungsverfahren durchlaufen. Voraussetzung zur Zulassung zu diesem Verfahren ist der Abschluss einer nach $\mathrm{BBiG} / \mathrm{HwO}$ oder Landesrecht geregelten mindestens zweijährigen Berufsausbildung und mindestens dreijähriger Berufspraxis in einem zum angestrebten Studium affinen Arbeitsbereich. Das Verfahren ist durch die jeweilige Hochschule auf der Grundlage der gültigen Prüfungsordnung durchzuführen. Es hat schriftliche und mündliche Prüfungsteile aufzuweisen und muss sowohl auf allgemeines als auch auf fachbezogenes Wissen ausgerichtet sein. Dieses Eignungsfeststellungsverfahren kann durch ein nachweislich erfolgreich absolviertes Probestudium im Umfang von mindestens einem Jahr ersetzt werden (vgl. ebd.).

Über diese bundeseinheitlichen Vorgaben der KMK hinaus obliegt es den einzelnen Bundesländern, weitergehende Regelungen für den Hochschulzugang zu treffen. Dies betrifft sowohl die Zulassung zu Hochschulprogrammen durch Anrechnung nachgewiesener Ausbildungsabschlüsse und berufspraktischer Erfahrungen als auch die Überprüfung vorhandener Kompetenzen mit dem Ziel des Hochschulzugangs durch eine gesonderte Prüfung. Dabei weisen die Hochschulgesetze der einzelnen Bundesländer große Schnittmengen auf. Um einen Überblick über die unterschiedlichen Ausgestaltungen auf landesrechtlicher Ebene geben zu können, werden einzelne Verfahren exemplarisch kurz dargestellt.

In Niedersachsen wird der Hochschulzugang für beruflich Qualifizierte im Landeshochschulgesetz geregelt. Hier ist besonders das sogenannte ,3+3-Verfahren“ zu nennen. Danach besitzt eine (fachgebundene) Hochschulzugangsberechtigung, wer 
nach Abschluss einer durch Bundes- oder Landesrecht geregelten mindestens dreijährigen Ausbildung in einem anerkannten Ausbildungsberuf in einem dem angestrebten Studiengang fachlich nahe stehenden Bereich diesen Beruf mindestens drei Jahre lang (...), ausgeübt hat (§ 18 Abs. 4 Sa. 2 Nr. 1 Niedersächsisches Hochschulgesetz - NHG).

Die fachlichen Zuordnungen der Ausbildungsberufe werden durch die jeweiligen Hochschulen vorgenommen. Viele Bundesländer, wie beispielsweise Hessen, Bayern und Nordrhein-Westfalen regeln den Hochschulzugang für beruflich Qualifizierte durch Rechtsverordnung. In diesem Rahmen werden beispielsweise in Hessen Steuerberaterinnen und -berater sowie Wirtschaftsprüfer und -prüferinnen mit dem Meisterabschluss gleichgesetzt und erhalten mit erfolgreichem Abschluss eine allgemeine HZB (vgl. Verordnung über den Zugang beruflich Qualifizierter zu den Hochschulen im Lande Hessen - BerQHSchulZV HE 2010). Auch zur Darstellung von Besonderheiten bei der Organisation der Hochschulzugangsprüfung kann das in Hessen praktizierte Verfahren herangezogen werden. Während in vielen Bundesländern, wie Thüringen oder Sachsen, die einzelnen Hochschulen die Prüfung selber durchführen, wurden in Hessen hochschulübergreifende Prüfungsausschüsse konstituiert, die jeweils für die Abnahme der Prüfungen in einem der 14 Studienbereiche für das gesamte Bundesland zuständig sind. Ein Ziel der Einrichtung dieser „Trägerhochschulen" ist es, aufgrund der Verteilung der fachlichen Zuständigkeiten eine Arbeitsteilung zu gewährleisten, so dass nicht jede Hochschule für alle angebotenen Fachrichtungen Ressourcen für die Durchführung der Hochschulzugangsprüfung vorhalten muss.

Weitestgehend unabhängig von den verschiedenen Regelungen und Ausgestaltungen einzelner Bundesländer folgt der Verfahrensablauf bundesweit einer einheitlichen Logik und kann daher generalisiert dargestellt werden. Dies ist darauf zurückzuführen, dass Verwaltungsakte in Deutschland unter Berücksichtigung des Verwaltungsverfahrensgesetzes ( VwVfG) durchzuführen sind. Daher folgen die Verfahren sowohl zur Zulassung zu Studienprogrammen als auch zur Verkürzung des zu studierenden Workloads unabhängig von landesrechtlichen Ausgestaltungen einer vergleichbaren Struktur.

Zunächst müssen die Bewerbenden einen Antrag auf Zulassung zu dem jeweiligen Studiengang beziehungsweise Prüfungsverfahren an die zuständige Hochschule richten. Die Teilnahme an solch einem Verfahren ist für Bewerbende in der Regel nicht mit zusätzlichen Prüfungsgebühren verbunden. Im Gegensatz zu dem Vorgehen in Frankreich erfolgt in Deutschland die Finanzierung des Verfahrens durch die Hochschulen selbst. Die antragprüfende Einrichtung definiert die für die Bearbeitung einzureichenden Unterlagen. Nach Einreichung durch die Antragstellenden werden diese auf Vollständigkeit überprüft und an den zuständigen Prüfungsausschuss beziehungsweise die zuständige Hochschulabteilung weitergeleitet. Handelt es sich um Abschlüsse gemäß der „Rahmenvereinbarung für Fachschulen“ (vgl. KMK 2014a) oder um Abschlüsse landesrechtlicher Fortbildungsregelungen (beispielsweise das ,3+3-Verfahren“ in Niedersachsen oder Abschlüsse, die nach der gesonderten Rechtsverordnung wie in Hessen einer HZB gleichgesetzt sind) erfolgt die direkte Zulassung zu dem Studienprogramm. Handelt es sich um einen Antrag auf Hochschulzugang durch eine gesonderte Prüfung, beschließt der Ausschuss über die Zulassung zur Prüfung. 
Die Hochschulzugangsprüfung wird auf der Grundlage einer Prüfungsordnung abgelegt. Ziel der Prüfung ist es festzustellen, ob Bewerbende in der Lage sind, das Studium mit Aussicht auf Erfolg aufzunehmen. Diese Prüfung soll in der Regel an die berufsbezogenen Erfahrungen der Bewerbenden anknüpfen und sowohl allgemeine als auch fachliche Grundlagen umfassen, die Voraussetzung für die Aufnahme des angestrebten Studiums sind. Wer die Prüfung bestanden hat, erhält ein Zeugnis über die Berechtigung, in einem entsprechenden Studienbereich an Hochschulen oder Berufsakademien in dem Bundesland zu studieren, in dem die Prüfung abgelegt wurde. Ein negative Überprüfung der Anträge hat schriftlich und durch Beifügen einer Rechtsmittelbelehrung an die Antragstellenden zu erfolgen.

Auch für die Zulassung zur Aufnahme eines Studiums in weiterbildenden Masterstudiengängen ohne HZB sowie ohne ersten berufsqualifizierenden Hochschulabschluss finden sich Regelungen in den unterschiedlichen Hochschulgesetzen der Länder. Die Verfahren entsprechen strukturell weitestgehend dem der Zulassung zu einem ersten berufsqualifizierenden Hochschulstudium. Darüber hinaus müssen Bewerbende im Rahmen einer Eignungsprüfung einen Kenntnisstand nachweisen, der dem eines für den angestrebten Studiengang einschlägigen ersten Hochschulabschlusses entspricht.

Neben durch die Hochschulen zu regelnden Prüfungen sehen die Auslegungshinweise der ländergemeinsamen Strukturvorgaben zur Akkreditierung von Bachelor- und Masterstudiengängen explizit auch die Anrechnung von außerhalb des Hochschulwesens erworbenen Kompetenzen und Fähigkeiten vor. Die Hoheit über die Ausgestaltung des Verfahrens obliegt hierbei der jeweiligen Hochschule.

\subsection{Vergleichende Darstellung der Verfahren zur Zulassung zu einem Studium ohne HZB}

Obwohl sich die Verfahren inhaltlich voneinander unterscheiden, wird in der Visualisierung deutlich, dass die Strukturen im Sinne des Verfahrensablaufs hohe Ähnlichkeiten aufweisen.

Wie Abb. 1 zeigt, werden die Unterschiede beim Blick auf die einzelnen Schritte deutlich. Während in Frankreich die außerhochschulisch erworbenen Kompetenzen auf die Äquivalenz zum Modulhandbuch des angestrebten Studiengangs hin verdeutlicht werden sollen, steht in Deutschland zunächst die Berücksichtigung von Berufsabschlüssen und entsprechender Berufserfahrung im Mittelpunkt. Dies kann auf die hohe Qualität und starke Strukturierung des dualen Ausbildungssystems in der Bundesrepublik zurückgeführt werden. Aus den in Kap. 2.2 genanntem Regelungen ergibt sich, dass durch den Nachweis von erfolgreich absolvierten und staatlich anerkannten Ausbildungen die Anschlussfähigkeit an Studienprogramme deutlich gemacht werden kann, ohne dass, wie in Frankreich, eine aufwendige zusätzliche Äquivalenzprüfung durchgeführt werden muss. Aber auch für den Hochschulzugang anhand einer Zulassungsprüfung gibt es in Deutschland Verfahren. Hier besteht der Nachteil, dass diese meist an jeder Hochschule unterschiedlich gestaltet werden und nur für das Bundesland gelten, in dem die Prüfung absolviert wurde. 
Frankreich (VAP)

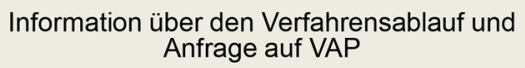
Anfrage auf VAP

Deutschland

Information über den Verfahrensablauf und Antragstellung auf Eignungsfeststellungsverfahren

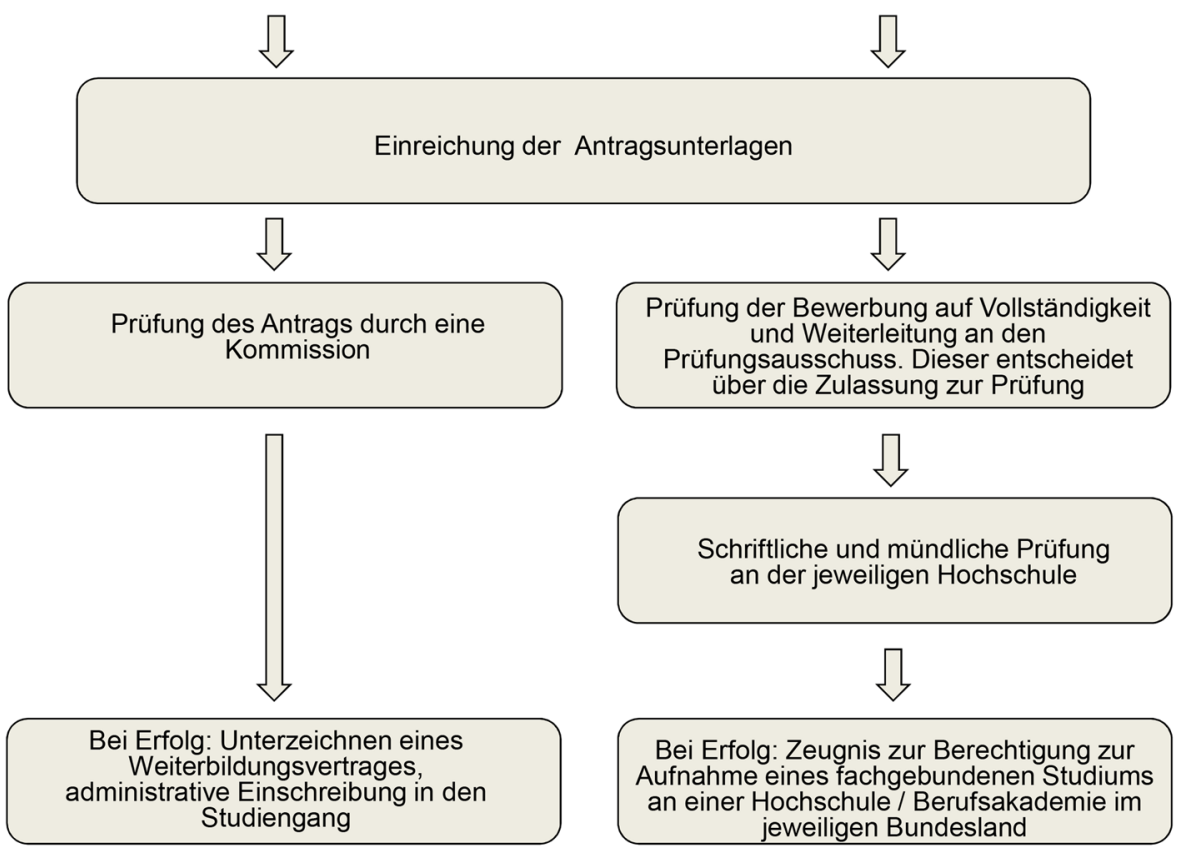

Abb. 1 Vergleich der Anrechnungsverfahren mit dem Ziel der Zulassung zu Studienprogrammen

\section{Vergleich der Systeme im Kontext der Verkürzung des zu studierenden Workloads}

Nachfolgend wird der Blick auf die Systematiken zur Verkürzung des zu studierenden Workloads mittels Anrechnung von außerhochschulisch erworbenen Kompetenzen gerichtet. Auch hier werden die Dimensionen Finanzen, Beratung und Vernetzung hervorgehoben, insofern sie innerhalb der Verfahren an Bedeutung gewinnen.

\subsection{Anrechnung als Instrument zur Reduktion des zu studierenden Workloads in Frankreich (VAE)}

Das Verfahren zur Anrechnung der außerhochschulisch erworbenen Kompetenzen VAE mit dem Ziel des Ersetzens von Studienleistungen ist im Verhältnis zur bereits dargestellten VAP komplexer und vielschichtiger (Abb. 1). So sind die potenziellen Antragstellenden darauf angewiesen, dass ihnen umfangreiche und transparente Informationen über das individuelle Verfahren generell und den operativen Prozess der Antragstellung zur Verfügung gestellt werden. Diesem Bedarf wird auf zwei unterschiedlichen Ebenen Rechnung getragen. Zum einen zentral, über staatlich 
geschaltete Internetseiten (www.vae.gouv.fr sowie www.vaeguidepratique.fr) und zum anderen im Rahmen der Internetauftritte der einzelnen Hochschulen, auf denen sowohl Informationen zum Verfahren an der Hochschule als auch die direkten Kontaktinformationen zu den jeweiligen VAE-Servicestellen, die jede französische Universität eigens für dieses Verfahren eingerichtet hat, hinterlegt sind. Die gesetzlichen Bestimmungen für die Anrechnung nach VAE sind in einer Verordnung von 2002 festgelegt (vgl. Französisches Bildungsministerium 2002).

Als Grundvoraussetzung, um das Verfahren auf Anrechnung außerhochschulisch erworbener Kompetenzen mit dem Ziel des Ersetzens eines gesamten Studienprogramms oder Teile desselben zu initiieren, müssen Bewerbende mindestens drei Jahre in einem auf den angestrebten akademischen Grad abzielenden Feld gearbeitet haben, unabhängig davon, ob ehrenamtlich oder als Arbeitnehmende.

Für Arbeitnehmende mit einer halben Stelle beträgt die nachzuweisende Stundenanzahl äquivalent 4800. Des Weiteren müssen das erworbene Wissen und die Fähigkeiten zum Teil oder vollständig mit den Anforderungen des jeweiligen Abschlusses übereinstimmen (vgl. Vereinigung VAE Guide Pratique 2013). Diese Prämissen zur Zulassung zu dem VAE-Verfahren sind in der Verordnung von 2002 im Artikel 2 festgelegt (vgl. Französisches Bildungsministerium 2002).

Erfüllen die an dem Verfahren interessierten Bewerbenden diese formalen Voraussetzungen, müssen sie zunächst eine Anfrage (la recevabilité) an die zuständige Stelle der jeweiligen Hochschule richten, an der eine Anrechnung vorgenommen werden soll. Dies ist notwendig, da sowohl jede Universität als auch jedes Studienfach das Vorgehen und die Beurteilung des VAE im Detail unterschiedlich gestalten.

Aufgrund der Zugangsvoraussetzungen zu dem Verfahren muss diese Anfrage neben dem Lebenslauf auch den Nachweis über die geforderten Arbeitszeiten enthalten. Dabei müssen die Bewerbenden einen Überblick darüber geben, welche Aufgaben sie während ihrer beruflichen Tätigkeiten absolviert haben und dies durch die Einreichung entsprechender Zeugnisse dokumentieren. Darüber hinaus muss im Rahmen des Antrags angegeben werden, welcher Hochschulabschluss (Zertifikat oder Studienabschluss) durch die Anrechnung der außerhochschulisch erworbenen Kompetenzen erlangt werden soll. Zur Vervollständigung der Antragsdokumente und der Zulassung zum Anrechnungsverfahren formulieren die Antragstellenden ihre Motivation für eine totale oder partielle Anrechnung ihrer Kompetenzen auf den jeweils angestrebten Abschluss.

In der zuständigen Abteilung der Hochschule werden die formalen Dokumente sowie die Erläuterungen zur jeweiligen Motivation überprüft und die Bewerbenden auf Basis dessen, insofern alle Prämissen erfüllt sind, entsprechend zu dem Anrechnungsverfahren zugelassen.

Erst nach der positiven Prüfung der Antragsdokumente und der Zulassung zum VAE durch die jeweilige Hochschule beginnt das eigentliche Verfahren zur Anrechnung.

Zunächst können sich die Bewerbenden einen Mentor oder eine Mentorin der universitären Behörde oder VAE Guide Pratique als Begleitung des Bewerbungsprozesses wählen. Die Finanzierung der Mentoren und Mentorinnen muss auch hier durch die Bewerbenden übernommen werden. Dieser oder diese unterstützt die Kandidatinnen und Kandidaten bei der Erstellung der Bewerbungsunterlagen, insbesondere bei der Beschreibung ihrer 
beruflichen Aktivitäten und bereits erworbenen Kompetenzen. Diese prozessbegleitende Beratung ist in der Regel fakultativ, an einigen Hochschulen jedoch obligatorisch.

Die Bewerbungsunterlagen (Dossier de validation) bestehen aus persönlichen und beruflichen Angaben. Ein zentraler Bestandteil für die Anrechnung ist der Nachweis und die detaillierte Beschreibung der außerhochschulisch erworbenen Kompetenzen und Arbeitstätigkeiten. Hierzu erhalten die Bewerbenden ein Muster als Vorlage. Die Aufgabe der Antragstellenden besteht darin, die Arbeitstätigkeiten so genau und detailliert wie möglich zu beschreiben. Dazu müssen sie die Unternehmen, in denen sie tätig waren, vorstellen und angeben, welchen Beruf sie dort ausgeübt haben. Zusätzlich hat eine Beschreibung von mindestens vier Arbeitstätigkeiten zu erfolgen. Als Grundlage für diese Beschreibung soll das Modulhandbuch des Hochschulprogramms dienen, auf das angerechnet werden soll. Um einen Äquivalenzvergleich der vorhandenen mit den anzurechnenden Kompetenzen zu ermöglichen, sollen sich die Bewerbenden bei der Darstellung ihrer außerhochschulisch erworbenen Kompetenzen an den Kompetenzbeschreibungen des Studienprogramms orientieren und die Äquivalenzen entsprechend verdeutlichen. Zur Unterstützung der Beschreibung und Darstellung der bereits erworbenen Kompetenzen sind Arbeitszeugnisse und belegende Dokumente der bisherigen Arbeitgeber von Vorteil (vgl. Vereinigung VAE Guide Pratique 2013). Die expliziten Anforderungen zum Inhalt der Bewerbungsunterlagen sind in der Verordnung des VAE von 2002 in Artikel 4 dokumentiert (vgl. Französisches Bildungsministerium 2002). Für diese Phase der Zusammenstellung der notwendigen Unterlagen beziehungsweise der Dokumentation der vorhandenen Kompetenzen haben die Antragstellenden in der Regel ein Jahr Zeit.

Nach dem Einreichen der Bewerbungsunterlagen erfolgt eine entsprechende Überprüfung durch eine Jury. Die Unterlagen dienen daraufhin als Basis für ein Interview vor dieser Jury (vgl. ebd.). Im Rahmen der Beratung der Bewerbenden unterstützen hier die VAE-Beratenden. Unter anderem bereiten sie gemeinsam mit den Bewerbenden das Interview vor. Die Jury wird vom Präsident der jeweiligen Hochschule bestimmt. Diese muss sich laut Artikel 5 aus einer Mehrheit von Lehrenden der Hochschule des angestrebten Studiengangs sowie aus Personen mit entsprechender Berufspraxis konstituieren. Dabei soll sie von Frauen und Männern paritätisch besetzt sein. Die Jurymitglieder sind so auszuwählen, dass sie über ausreichende Kenntnisse der geforderten Qualifikationen, Kompetenzen und des notwendigen Wissens des Kandidaten oder der Kandidatin verfügen.

Im Rahmen des Interviews erfolgt zunächst eine zehn- bis 15-minütige Vorstellung der Bewerbenden zu Person und beruflichem Werdegang. Daraufhin werden eventuell auftretende Unklarheiten in den Bewerbungsunterlagen durch die Jury aufgelöst und die Antragstellenden werden bezüglich ihres theoretischen Wissens und ihrer Kompetenzen befragt. Bei einzelnen Hochschulen ist es vorgesehen, dass die Kandidatinnen und Kandidaten im Rahmen der Interviews die Lösung eines Problems aus der Berufspraxis antizipieren (vgl. ebd.). Unter Berücksichtigung der Bewerbungsunterlagen und dem Gespräch mit den Bewerbenden entscheidet die Jury über die totale oder partielle Anrechnung oder die Ablehnung der außerhochschulisch erworbenen Kompetenzen auf das Studienprogramm. Dabei ist die Jury dazu verpflichtet, ihre Entscheidung über die Anrechnung neutral, objektiv und ohne jegliche Form von Diskriminierung zu treffen (vgl. Interministerielles Komitee für die Entwicklung von VAE 2009). 
Jede Jury definiert die konkreten Details der Kriterien für die Entscheidungsfindung selbst. Dabei gibt es als Hilfestellung und Orientierung eine vorstrukturierte Übersicht über die Kriterien der Validierung. Darin ist definiert, dass sich die Mitglieder an dem beruflichen Werdegang der Bewerbenden orientieren sollen, da dies ein zentraler Indikator für die vorhandenen theoretischen und praktischen Kompetenzen ist und somit als Grundlage für einen Äquivalenzvergleich herangezogen werden kann. Hierbei steht die kritische Begutachtung der Beispiele aus der Erwerbstätigkeit im Fokus. Um die von den Bewerbenden angegebenen Kompetenzen zu überprüfen, wird im Rahmen des Interviews abgefragt, ob sie hinreichend über Kompetenzen wie wissenschaftliches Argumentieren, Analysieren, Problemantizipation und die entsprechende Methodik in der jeweiligen Theorie und Praxis verfügen.

Kommt die Jury nach dem Interview abschließend zu dem Ergebnis, dass keine vollständige, sondern lediglich eine partielle Anrechnung ausgesprochen werden kann, erhalten der Bewerbenden die Möglichkeit, sich durch weitere fortbildende Maßnahmen zu einem späteren Zeitpunkt erneut für die Anrechnung des gesamten Studienprogramms durch das VAE zu bewerben (vgl. Vereinigung VAE Guide Pratique 2013). Die Zulassung zum VAE kann für einen Studienabschluss lediglich einmal pro Jahr gestellt werden. Insgesamt können für verschiedene Abschlüsse bis zu drei Anfragen eingereicht werden (vgl. Französisches Bildungsministerium 2002). Des Weiteren ist festzuhalten, dass in Frankreich immer ein individuelles Verfahren zur Anrechnung gewählt wird. Die Möglichkeit einer pauschalen Anrechnung von außerhochschulisch erworbenen Kompetenzen scheint hier nicht vorgesehen. Interessant wäre, in einem weiteren Schritt zu prüfen, ob der Weg vom individuellen zum pauschalen Verfahren (vgl. Loroff et al. 2011, S. 113) in Frankreich prinzipiell möglich wäre.

Das gesamte Verfahren dauert in der Regel zwischen sechs und zwölf Monaten. Die Verfahrenskosten belaufen sich, ohne die Betreuung durch einen Mentor oder eine Mentorin, je nach Hochschule auf 600 bis $2000 €$ und sind vollständig von den Antragstellenden zu tragen. Werden VAE-Beratende zur Unterstützung rekrutiert, steigen die Kosten je nach Umfang und Aufwand der zu erbringenden Leistung entsprechend. Es bestehen jedoch Möglichkeiten zur Finanzierung des Verfahrens (vgl. Vereinigung VAE Guide Pratique 2013).

\subsection{Anrechnung als Instrument zur Reduktion des zu studierenden Workloads in Deutschland}

In der föderalistisch strukturierten deutschen Bildungslandschaft besteht kein bundesweit genormtes oder gerahmtes Verfahren zur Anrechnung außerhochschulisch erworbener Kompetenzen mit dem Ziel der Verkürzung des zu studierenden Workloads. Die KMK hat im Jahr 2002 lediglich definiert, dass maximal 50\% außerhochschulisch erworbener Kompetenzen auf ein Studienprogramm anrechenbar sind (vgl. KMK 2002). Diese Regelung wurde mit unterschiedlichen Formulierungen in die Landeshochschulgesetze übernommen. Während das Hessische Hochschulgesetz vorgibt, dass außerhalb von Hochschulen erworbene Kompetenzen mit bis zu 50\% auf ein Hochschulstudium angerechnet werden „können“ (§ 18 Abs. 6 HHG), findet sich beispielsweise im Brandenburgischen und Baden-Württembergischen Hoch- 
schulgesetz die Formulierung, dass diese Kompetenzen bei der Feststellung von Gleichwertigkeit ,anzurechnen sind“ ( 24 Abs. 5 Brandenburgisches Hochschulgesetz - BbgHG, § 35 Abs. 3 Gesetz über die Hochschulen in Baden-Württemberg $-\mathrm{LHG})$.

Weitestgehend einheitlich in den Landeshochschulgesetzen sind die Kriterien definiert, die erfüllt sein müssen, damit eine Anrechnung außerhochschulisch erworbener Kompetenzen möglich ist. So müssen die Kompetenzen gleichwertig zu den zu ersetzenden Studieninhalten sein. Darüber hinaus sind die Kriterien für die Anrechnung im Rahmen der Akkreditierung zu überprüfen. Mit welchen Verfahren und anhand welcher Instrumente die geforderte Gleichwertigkeit im Einzelnen festzustellen ist, wird nicht weiter definiert und obliegt den jeweiligen Hochschulen. Die Finanzierung dieser Verfahren wird in der Regel von den Hochschulen gewährleistet, so dass den Kandidatinnen und Kandidaten keine Mehrkosten entstehen. Des Weiteren wurde die Verfahrenshoheit der Hochschulen auf diesem Gebiet auch 2008 durch die KMK noch einmal hervorgehoben.

Die Hochschulen sind Garant für die Qualität der von ihnen verliehenen Hochschulabschlüsse und -grade. Sie sind verantwortlich für die Qualitätssicherung der Studienprogramme sowie der Anrechnungsverfahren nach Ziffer 2. Dies setzt voraus, dass ein wesentlicher Teil der dem Hochschulabschluss zugrundeliegenden Ausbildung in der unmittelbaren Verantwortung, d. h. durch eigene Leistungen der verleihenden Hochschule stattfindet (KMK 2008).

Dies zeigt, dass die vollständige Anrechnung ganzer Studienprogramme auf Grundlage außerhochschulisch erworbener Kompetenzen in Deutschland nicht zulässig ist. In den vergangenen zehn Jahren wurden im Rahmen der ANKOM-Initiative und des Bundeswettbewerbs Offene Hochschulen - Aufstieg durch Bildung! vielfältige Instrumente zur Kompetenzbilanzierung sowie der individuellen, pauschalen und kombinierten Anrechnung außerhochschulisch erworbener Kompetenzen entwickelt und erprobt. Dennoch haben sie sich bis heute in der deutschen Hochschullandschaft nicht in der Breite durchsetzen können. Ebenso wenig gibt es an jeder Hochschule Beratungsstellen, die sich ausschließlich mit der Anrechnung außerhochschulisch erworbener Kompetenzen beschäftigen. Daher soll die abschließende Identifikation von Adaptierungspotenzialen einzelner Instrumente des französischen Systems Anregungen dazu geben, welche Rahmenbedingungen die nachhaltige Implementierung der bereits in Deutschland entwickelten Verfahren begünstigen könnten.

\subsection{Vergleichende Darstellung der Verfahren zur Verkürzung des zu studierenden Workloads}

Während das Verfahren zur Anrechnung von Kompetenzen auf Studienangebote in Frankreich landesweit vereinheitlicht und klar definiert ist, lassen sich in Deutschland sowohl zwischen den Bundesländern als auch zwischen den Hochschulen innerhalb der einzelnen Bundesländer verschieden ausgeprägte Systematiken beobachten. Demnach kann sich hier bei dem Versuch einer bundeseinheitlichen Systematisierung des Verfahrens lediglich an den relevanten Aspekten im Verwaltungsverfahrensrecht orientiert werden (vgl. Hanak und Sturm 2015a). Zudem zeichnet sich das 
französische System sehr stark durch den Beratungs- beziehungsweise Begleitungsaspekt der VAE-Beratenden aus: Beginnend mit nationalen sowie hochschuleigenen Informationsseiten im Internet, über VAE-Beratungsstellen an jeder französischen Hochschule bis hin zu Mentorinnen und Mentoren, welche die Bewerbenden über das gesamte Verfahren hinweg unterstützen. Wie schon bei der Anrechnung mit dem Ziel der Zulassung zu Studienprogrammen, profitieren die französischen Hochschulen auch hier von zwei zentralen Aspekten. Zum einen bestehen national einheitliche Vorgaben zur Durchführung der Anrechnung und zum anderen werden die Kosten für die entsprechenden Verfahren an die Bewerbenden weitergegeben.

Abbildung 2 macht deutlich, dass auch hier, wie schon bei der grafischen Darstellung des Anrechnungsverfahrens mit dem Ziel der Zulassung zu einem Studienprogramm, eine hohe Vergleichbarkeit der einzelnen Verfahrensschritte zwischen den beiden Ländern vorliegt. In Deutschland räumt der Gesetzgeber den Hochschulen einen großen Spielraum zur Ausgestaltung der Anrechnungsverfahren ein. Daher ist zu vermuten, dass hier ungenutzte Adaptierungspotenziale identifiziert werden können, die einen Beitrag dazu leisten, belastbare Verfahren nachhaltig zu implementieren.

\section{Adaptierungspotenziale für das deutsche Hochschulsystem}

Mit der Darstellung der Verfahren wird deutlich, welche umfangreichen Möglichkeiten und Konzepte zur Anrechnung außerhochschulisch erworbener Kompetenzen sowohl in Frankreich als auch in Deutschland bereits vorliegen. Im Folgenden werden potenzielle Adaptierungspotenziale anhand der eingangs formulierten Faktoren „Beratung“, „Finanzierung“ und „Vernetzung" herausgearbeitet. Dabei soll der Blick explizit auf die entsprechenden Schwächen im deutschen System gerichtet werden.

In der Gesamtschau zeigt sich, dass insbesondere für das VAE die Beratung der Antragstellenden einen hohen Stellenwert im französischen System hat. Bei einer so komplexen Aufgabe wie der Kompetenzbilanzierung mit dem Fokus auf der Darstellung von Äquivalenten zu den Lernergebnissen von Studienprogrammen, benötigen Antragstellende die Unterstützung von Expertinnen und Experten. Die umfassende und prozessbegleitende Beratung der VAE-Beratenden an den französischen Hochschulen leistet einen zentralen Beitrag dazu, die Dokumentation der außerhochschulisch erworbenen Kompetenzen transparent und qualitativ hochwertig sicherzustellen. Eine flächendeckende und an jeder Hochschule fest zu installierende Beratungsstelle könnte auch im deutschen Hochschulsystem besonders bei der Anrechnung mit dem Ziel zur Verkürzung des zu studierenden Workloads einen wichtigen Beitrag leisten, um die Hochschulen für nicht traditionell Studierende zu öffnen. Das Sichtbarmachen von Kompetenzen als Grundlage für einen Äquivalenzvergleich mit den Lernergebnissen im Modulhandbuch des Studienprogramms, auf das angerechnet werden soll, ist eine hoch anspruchsvolle Aufgabe, die ohne Weiteres weder von den Bewerbenden noch den inhaltlich verantwortlichen Lehrenden übernommen werden kann.

Ein mit der Forderung nach Beratung eng verbundener Aspekt ist die Frage nach dem finanziellen Aufwand für die Hochschulen. In Frankreich sind die Kos- 
Frankreich (VAE)

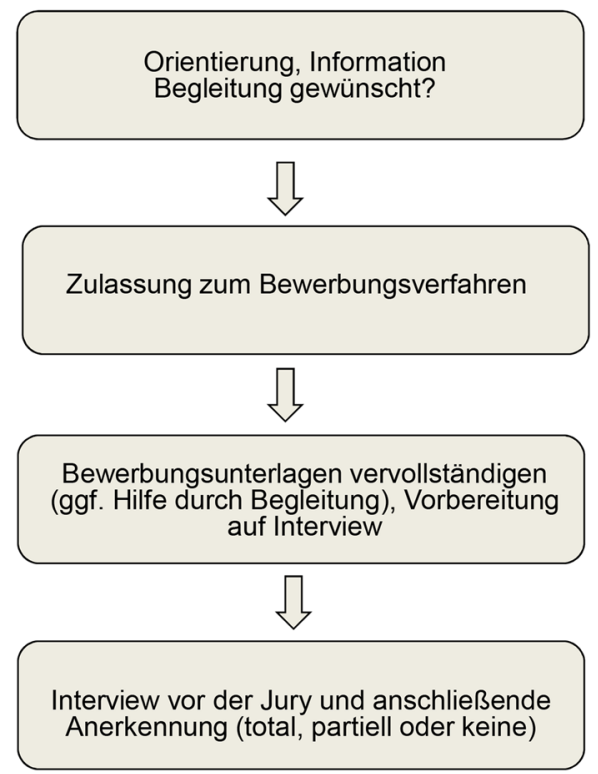

Deutschland

Orientierung, Recherche, Information (da an jeder deutschen Hochschule unterschiedlich und nicht verpflichtend)

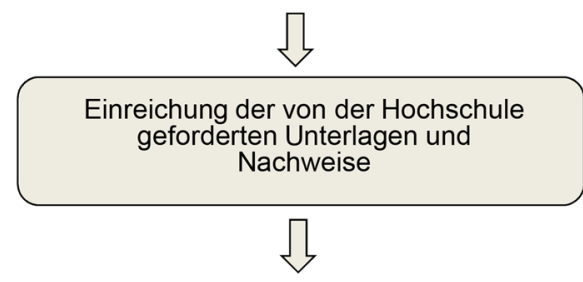

Prüfung der Unterlagen durch die zuständige Stelle der Hochschule

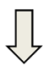

Ggf. Anrechnung von bis zu max. 50 Prozent des zu studierenden Workloads

Abb. 2 Vergleich der Anrechnungsverfahren mit dem Ziel der Reduzierung des zu studierenden Workloads

ten, sowohl für die allgemeine Verfahrensabwicklung als auch für die individuelle Beratungsleistung, von den Bewerbenden zu tragen. Eine Adaptierung dieses Konzepts an deutschen Hochschulen könnte die Akzeptanz für Verfahren der Anrechnung außerhochschulisch erworbener Kompetenzen innerhalb der Hochschulen stärken. Denn die durch die Entwicklung und Durchführung solcher Verfahren entstehenden Kosten stellen ein zentrales Hindernis für die Einführung dar (vgl. Hanak und Sturm 2015b). Durch die vollkostendeckende Übernahme seitens der Bewerbenden entfällt das finanzielle Risiko für die Hochschulen. Damit kann sich die Bereitschaft erhöhen, sich solchen neuartigen Serviceleistungen zu öffnen, zumal sie auch einen Wettbewerbsvorteil zur Erschließung neuer Zielgruppen für die jeweilige Hochschule bedeuten kann. Um zu vermeiden, dass die Teilhabe an einem solchen Verfahren von den finanziellen Möglichkeiten der einzelnen Antragstellenden abhängt, wäre es notwendig, dass staatliche Förderprogramme die Kosten für das Verfahren ganz oder anteilig übernehmen können, so wie es auch in Frankreich möglich ist.

Gerade auf der Grundlage der föderalen Struktur im Bildungssystem der Bundesrepublik erscheint die Organisation eines engen Austauschs der Expertinnen und Experten untereinander als zentrale Herausforderung. Hier gilt es, Instrumente der Vernetzung zwischen den Hochschulen über (Bundes-)Ländergrenzen hinweg zu entwickeln. Durch das Zusammenführen der Erfahrungen und des Wissens des Fachpersonals an den jeweiligen Universitäten können Prozesse optimiert und die verschiedenen Erfahrungen landesweit nutzbar gemacht werden. Auch eine solche nationale Vernetzung der Akteure und Akteurinnen untereinander mit dem gemeinsamen Ziel, die Verfahren weiter zu optimieren, birgt Adaptionspotenzial für die deutsche Hochschullandschaft. Hiermit könnte ein relevanter Beitrag dazu geleistet 
werden, die als Ergebnisse aufwendiger Forschungs- und Entwicklungsarbeiten in den vergangenen zehn Jahren im gesamten Bundesgebiet entstandenen Verfahren nachhaltig über die entwickelnden Hochschulen hinaus zu implementieren.

Auch wenn aufgrund der KMK-Vorgaben, dass maximal 50\% außerhochschulisch erworbene Kompetenzen auf ein Hochschulprogramm angerechnet werden dürfen, und dass ein wesentlicher Teil der dem Hochschulabschluss zugrunde liegenden Ausbildung in der unmittelbaren Verantwortung der Hochschule stattfindet, eine vollständige Adaptierung der französischen Verfahren allein aus rechtlicher Perspektive ausgeschlossen ist, erscheint die zugrunde gelegte Systematik, gerade im Hinblick auf die Beratungsleistungen, die Kostenverteilung und den Aspekt der Vernetzung hinreichend Anknüpfungspunkte zur Optimierung der Verfahren an deutschen Hochschulen zu bieten. Dies könnte auch einen Beitrag dazu leisten, dass die in Deutschland bereits bestehenden Möglichkeiten zur Anrechnung offensiver beworben werden.

Open Access Dieser Artikel wird unter der Creative Commons Namensnennung 4.0 International Lizenz (http://creativecommons.org/licenses/by/4.0/deed.de) veröffentlicht, welche die uneingeschränkte Nutzung, Verbreitung und Wiedergabe für beliebige Zwecke erlaubt, sofern Sie den/die ursprünglichen Autor(en) und die Quelle ordnungsgemäß nennen, einen Link zur Creative Commons Lizenz beifügen und angeben, ob Änderungen vorgenommen wurden.

\section{Literatur}

Französisches Bildungsministerium. (1985). Décret VAP 1985. Décret $n^{\circ} 85-906$ du 23 août 1985 fixant les conditions de validation des études, expériences professionnelles ou acquis personnels en vue de l'accès aux différents niveaux de l'enseignement supérieur. www.legifrance.gouv.fr/affichTexte.do;jsessionid=AA3C26DA5258D11781326DA02651DA81.tpdjo13v_3?cidTexte=JORFTEXT000000689033\&dateTexte=20111010. Zugegriffen: 17. Mai 2015.

Französisches Bildungsministerium. (2002). Décrets, arrêtés, circulaires. Textes généraux Ministère de l'éducation nationale. Décret VAE 2002. Unter Mitarbeit von Lionel Jospin, Jack Lang und Élisabeth Guigou. www.utc.fr/vae/pages/decrets/decret_VAE.pdf. Zugegriffen: 03. Juni 2015.

Freitag, W. K., Buhr, R., Danzeglocke, E., Schröder, S., \& Volk, D. (Hrsg.). (2015). Übergänge gestalten. Durchlässigkeit zwischen beruflicher und hochschulischer Bildung erhöhen. Münster: Waxmann.

Hanak, H., \& Sturm, N. (2015a). Anerkennung und Anrechnung außerhochschulisch erworbener Kompetenzen. Eine Handreichung für die wissenschaftliche Weiterbildung. Wiesbaden: Springer VS.

Hanak, H., \& Sturm, N. (2015b). Außerhochschulisch erworbene Kompetenzen anrechnen. Praxisanalyse und Implementierungsempfehlungen. Wiesbaden: Springer VS.

Interministerielles Komitee für die Entwicklung von VAE. (2009). Charte de déontologie des membres de jury de validation des acquis de l'expérience. www.vaeguidepratique.fr/Dossier\%20Image/telechargement/VAE_deontologie_jury.pdf. Zugegriffen: 05. Juni 2015.

KMK - Konferenz der Kultusminister der Länder in der Bundesrepublik Deutschland. (2002). Anrechnung von außerhalb des Hochschulwesens erworbenen Kenntnissen und Fähigkeiten auf ein Hochschulstudium (I). (Beschluss der Kultusministerkonferenz vom 28.06.2002). www.kmk.org/fileadmin/veroeffentlichungen_beschluesse/2002/2002_06_28-Anrechnung-Faehigkeiten-Studium-1. pdf. Zugegriffen: 05. Juni 2015 .

KMK - Konferenz der Kultusminister der Länder in der Bundesrepublik Deutschland. (2008). Anrechnung von außerhalb des Hochschulwesens erworbenen Kenntnissen und Fähigkeiten auf ein Hochschulstudium (II). (Beschluss der Kultusministerkonferenz vom 18.09.2008). www.akkreditierungsrat.de/ fileadmin/Seiteninhalte/KMK/Vorgaben/KMK_Anrechnung_ausserhochschulisch_II.pdf. Zugegriffen: 05. Juni 2015. 
KMK - Konferenz der Kultusminister der Länder in der Bundesrepublik Deutschland. (2009). Hochschulzugang für beruflich qualifizierte Bewerber ohne schulische Hochschulzugangsberechtigung. (Beschluss der Kultusministerkonferenz vom 06.03.2009). www.kmk.org/fileadmin/veroeffentlichungen_beschluesse/2009/2020_03_06-Hochschulzugang-erful-qualifizierte-Bewerber.pdf. Zugegriffen: 02 . Juni 2015.

KMK - Konferenz der Kultusminister der Länder in der Bundesrepublik Deutschland. (2011). Ländergemeinsame Strukturvorgaben für die Akkreditierung von Bachelor- und Masterstudiengängen vom 04.02.2010. Anlage 1: Auslegungshinweise vom 25.03.2011. http://www.pst.kit.edu/download/ Strukturvorgaben_Auslegungshinweise_kmk_mai_2011.pdf. Zugegriffen: 19. Nov. 2015.

KMK - Konferenz der Kultusminister der Länder in dēr Bundesrepublik Deutschland. (2014a). Rahmenvereinbarung über Fachschulen. (Beschluss der Kultusministerkonferenz vom 07.11.2002 i.d.F. vom 25.09.2014). www.kmk.org/fileadmin/veroeffentlichungen_beschluesse/2002/2002_11_07-RVFachschulen.pdf. Zugegriffen: 21. Juni 2015.

KMK - Konferenz der Kultusminister der Länder in der Bundesrepublik Deutschland. (2014b). Synoptische Darstellung der in den Ländern bestehenden Möglichkeiten des Hochschulzugangs für beruflich qualifizierte Bewerber ohne schulische Hochschulzugangsberechtigung auf der Grundlage hochschulrechtlicher Regelungen (Stand August 2014). www.kmk.org/fileadmin/veroeffentlichungen beschluesse/2014/2014_08_00-Synopse-Hochschulzugang-berufl_Qualifizierter.pdf. Zugegriffen: 19. Nov. 2015.

Loroff, C., Stamm-Riemer, I., \& Hartmann, E. A. (2011). Anrechnung: Modellentwicklung, Generalisierung und Kontextbedingungen. In W. K. Freitag, E. A. Hartmann, C. Loroff, I. Stamm-Riemer, D. Völk, \& R. Buhr (Hrsg.), Gestaltungsfeld Anrechnung. Hochschulische und berufliche Bildung im Wandel (S. 77-117). Münster: Waxmann.

Schäfer, M., Kriegel, M., \& Hagemann, T. (Hrsg.). (2015). Neue Wege zur akademischen Qualifizierung im Sozial- und Gesundheitssystem. Berufsbegleitend studieren an Offenen Hochschulen. Münster: Waxmann.

Seger, M. S., \& Waldeyer, C. (2015). Qualitätssicherung im Kontext der Anrechnung und Anerkennung von Lernergebnissen an Hochschulen. Standards für transparente und nachvollziehbare Analyseverfahren und Anrechnungsprozesse. Aachen: Shaker.

Vereinigung VAE Guide Pratique. (2013). VAE Guide Pratique. www.vaeguidepratique.fr/index.htm. Zugegriffen: 05. Juni 2015. 